\title{
Drei-Klassen-Gesellschaft? Bruch? Konfrontation? Eine Auseinandersetzung mit Andreas Reckwitz' Diagnose der »Spätmoderne«
}

"Die Menschen hier sehen alle gleich aus, irgendwie individuell. ${ }^{1}$

\section{Einleitung}

Andreas Reckwitz' Studie zur Gesellschaft der Singularitäten ${ }^{2}$ gehört zu den in der Öffentlichkeit meistbeachteten deutschsprachigen soziologischen Zeitdiagnosen. Mit seinem neuesten Buch über Das Ende der Illusionen ${ }^{3}$ baut er seine Position des exponierten öffentlichen Intellektuellen weiter aus: In allen großen Tageszeitungen wurde das Buch besprochen, ${ }^{4}$ seine Konzepte dienen mittlerweile als Stichworte in den politischen Debatten des Feuilletons ${ }^{5}$ - wenn er nicht gleich selbst mit Beiträgen und Interviews zur Lage der Dinge zu Wort kommt. ${ }^{6}$ Mehr noch: Verschiedene Bundespolitikerinnen und -politiker aus unterschiedlichen Parteien bezogen in Videokonferenzen und auf ihren Instagram-Accounts das signalorange leuchtende Buch in ihr »impression management ${ }^{7}$ ein. ${ }^{8}$ Sogar die sonst nicht gerade für ihre Rezensionen soziologischer Fachliteratur bekannte $B I L D$-Zeitung berichtete darüber und versprach, »Das Geheimnis hinter diesem Buch « - so der Titel des Artikels - zu lüften. ${ }^{9}$

Ohne Frage teilt Reckwitz' Buch die von ihm selbst an anderer Stelle herausgestellten Merkmale einer erfolgreichen, zeitgenössischen intellektuellen Intervention: Es ist eine "großflächige, pointierte Problemanalyse [...] mit einem Schuss engagierter Unberechenbarkeit ${ }^{10}{ }^{10}$. Es sind vor allem zwei Aspekte seiner Zeitdiagnose, die in den zahlreichen Rezensionen hervorgehoben werden. Zum einen

1 Rainald Grebe: »Prenzlauer Berg «, 2011 (Songtext).

2 Reckwitz 2017 a.

3 Reckwitz 2019 a.

4 Zum Beispiel Grau 2019; Nassehi 2020; Sobocynski 2020; Wohlfahrt 2020.

5 Zum Beispiel Charim 2018; Pausch 2020; Sauerbrey 2020.

6 Zum Beispiel Teuwsen 2020; Reinecke 2020; Reckwitz 2020.

7 Goffman 1959.

8 Balzter et al. 2020.

9 Schütz, Schuler 2020.

10 Reckwitz 2017 b. 
handelt es sich wirklich um eine umfassende Zeitdiagnose - ein Krisenpanorama, das nicht weniger als einen Epochenbruch konstatiert und beansprucht, als »Ordnungsruf «11 aufzuzeigen, welche entscheidenden Weichen für die Zukunft jetzt gestellt werden müssten. ${ }^{12}$ Zum anderen verortet Reckwitz den Bruch, um den es ihm geht, im Herzen der Mittelschichten, als eine sich in zwei Lager aufspaltende Konfrontation: zwischen der aufsteigenden "neuen Mittelklasse", die sich dem wirtschaftlichen und kulturellen Liberalismus verschrieben habe und deshalb verständnislos oder ablehnend der anderen, absteigenden »alten Mittelklasse " gegenüberstehe, die sich in Reaktion nun trotzig dem Rechtspopulismus zuwende. Dieser Bruch drohe die Gesellschaft zu zerreißen.

Reckwitz liefert damit einen empirisch greifbaren und intuitiv plausiblen Schlüssel zu den politischen Verwerfungen der letzten Dekade. Mit anderen Worten: Dass das Buch als "public sociology «13 so dankbar aufgenommen wird, liegt wohl genau daran, dass die darin unterbreitete Sicht der Dinge als »Augenöffner " erscheint - eine stimmige und viele Rätsel beantwortende Analyse. Die Kernthesen von Reckwitz greifen auf und beeinflussen zugleich, wie die gegenwärtige deutsche Gesellschaft sich selbst wahrnimmt und ihre Probleme bearbeitet. Das ist Grund genug, soziologisch genauer hinzuschauen. Hinzu kommt, dass Reckwitz' Diagnose einen Beitrag zu einem Strang des umfassenderen Diskurses über die Perspektiven der Mittelschichten in den entwickelten westlichen Gesellschaften darstellt, der in den letzten Jahren von einer ganzen Reihe soziologischer Zeitdiagnosen mitgeprägt worden ist. ${ }^{14}$

Darum geht es also im Folgenden: kritisch zu prüfen, wie stichhaltig Reckwitz' Diagnose ist. Weil Das Ende der Illusionen erst vor gut einem Jahr veröffentlicht wurde, gibt es hierzu noch keine eingehende fachliche Diskussion. ${ }^{15}$ Wir wollen diese Diskussion hier initiieren - und zwar im Kern mit einer Auseinandersetzung mit dem dritten Kapitel »Von der nivellierten Mittelstandsgesellschaft zur DreiKlassen-Gesellschaft: Neue Mittelklasse, alte Mittelklasse, prekäre Klasse«. Dieses Kapitel ist dafür besonders geeignet, weil - hic rhodus, hic salta - die dortige Konkretisierung und Operationalisierung der Gesellschaftsdiagnose eine empirisch grundierte Überprüfung erlaubt. Dabei können wir Befunde aus unserem

\section{Reckwitz 2019b.}

12 Wobei er aber - und das ist mit Blick auf eine möglichst breite Rezeption strategisch klug - uneindeutig genug bleibt, sodass etwa Friedrich Merz reklamieren kann, diese von Reckwitz geforderte Gesellschaft der Zukunft sei eben die altvertraute »soziale Marktwirtschaft « (Schlieben 2020).

13 Buroway 2005.

14 Für den deutschsprachigen Raum siehe unter anderem Mau 2012; Nachtwey 2016; Koppetsch 2019; für den anglophonen Raum etwa Fraser 2017; Hochschild 2017 [2016].

15 Anders als zum Vorgängerbuch über Die Gesellschaft der Singularitäten. Ausnahmen sind die bereits erwähnte Kritik Armin Nassehis (2020) sowie eine Rezension Ulrich Bröcklings (2019). Beide konzentrieren sich jedoch gerade nicht auf die empirischen Aussagen des Buchs, was wir für unverzichtbar halten, auch um die theoretische Konstruktion des Gesellschaftsmodells zu beurteilen. 
kürzlich abgeschlossenen Forschungsprojekt »Lebensführung als investive Statusarbeit « 16 einbringen, die dort aus biografisch-narrativen Interviews mit Angehörigen verschiedener Einkommens-, Bildungs- und Alterssegmente der deutschen Mittelschichten hervorgehen.

Im nächsten Abschnitt arbeiten wir Reckwitz' Kernthesen heraus. Die drei darauf folgenden Abschnitte unterziehen diese Kernthesen einer Drei-KlassenGesellschaft, eines Bruchs gegenüber der vorherigen »nivellierten Mittelstandsgesellschaft « und einer zunehmenden Konfrontation zwischen einer »neuen « und einer »alten Mittelklasse" dann einer eingehenden Prüfung und heben hervor, wo es Uneindeutigkeiten, Widersprüche und Übervereinfachungen in der konzeptionellen Anlage des theoretischen Modells gibt. Dem stellen wir empirische Befunde sowie theoretische Überlegungen anderer Autorinnen und Autoren sowie einige Beobachtungen aus unserer Studie gegenüber, die uns zu der gegenüber Reckwitz entdramatisierenden Schlussfolgerung geführt haben, dass wir es in Deutschland wohl nicht mit einer antagonistisch gespaltenen Mittelschicht zu tun haben. Auch wenn das erst einmal so klingen mag, als gössen wir jede Menge Wasser in den von Reckwitz gekelterten Wein: Unsere Absicht ist nicht, eine in der Tat neue und möglicherweise wegweisende Perspektive einfach vom Tisch zu wischen. Wir wollen vielmehr auf - zugegeben: zahlreiche - theoretische und empirische Stolpersteine aufmerksam machen, damit Reckwitz und andere, die aus vergleichbaren Perspektiven zu denselben Fragen arbeiten, an diesen Punkten neu ansetzen können. Doch selbst wenn sich dabei herausstellen sollte, dass das vorgeschlagene Modell gar nicht aufrechtzuerhalten ist, bliebe es Reckwitz' unbestreitbares Verdienst, durch sein Angebot die Aufmerksamkeit auf Problemkomplexe gelenkt zu haben, die auch ganz anders ausgerichtete Analysen zum Zusammenhang von kulturellem und sozialstrukturellem Wandel auf der einen, gesellschaftspolitischen Konflikten auf der anderen Seite bewältigen müssten.

\section{Theoriearchitektur}

Anhaltspunkte dafür, warum Reckwitz für viele »den Nerv der Zeit « trifft, haben wir uns vergegenwärtigt. Nun gilt es zu prüfen: Liefert Reckwitz wirklich, was er verspricht? Hierfür muss man sich zunächst sehr knapp - die einschlägige Belesenheit voraussetzend - vergegenwärtigen, worin der theoretische Kern seiner Gegenwartsdiagnose besteht. Die von Reckwitz angebotene Diagnose kombiniert

16 Leitung: Karin Gottschall, Betina Hollstein und Uwe Schimank; Mitarbeiter: Nils Kumkar und Stefan Holubek; Laufzeit: Oktober 2016 bis Oktober 2019; Förderung: Deutsche Forschungsgemeinschaft. 
eine kultur- und eine ungleichheitstheoretische ${ }^{17}$ Perspektive auf die "Spätmoderne ${ }^{18}{ }^{18}$, die sich in drei Kernthesen verdichten lässt:

- Die aktuelle Gesellschaft der Bundesrepublik lässt sich als eine Drei-KlassenGesellschaft aus "alter Mittelklasse«, "neuer Mittelklasse« und »prekärer Klasse " beschreiben.

- Diese gegenwärtige gesellschaftliche Situation stellt einen sich seit Mitte der 1970er Jahre vollziehenden und immer weiter vertiefenden Bruch gegenüber dem gesellschaftlichen Zustand dar, der vor dieser Zeit herrschte.

- Der Bruch besteht darin, dass sich seitdem eine neue Linie der Konfrontation aufgetan hat - zwischen der » alten « und der »neuen Mittelklasse«.

Reckwitz ${ }^{19}$ macht hierfür drei gesellschaftliche Dynamiken als Treiber verantwortlich:

- Postindustrialisierung: Die kapitalistische Wirtschaft in den entwickelten westlichen Gesellschaften wird immer weniger durch große und arbeitsintensive Industriebranchen und immer stärker durch Dienstleistungsbranchen der "Wissensarbeit « ${ }^{20}$ geprägt.

- Bildungsexpansion: Der Anteil derjenigen, die einen akademischen Abschluss erwerben, ist unaufhörlich gestiegen, sodass sie erstmals nicht nur einen sehr kleinen, sondern einen beträchtlichen, in manchen Ländern zumindest in den jüngeren Kohorten sogar schon den größten Bevölkerungsanteil ausmachen.

- Wertewandel: An die Stelle des kulturell hegemonialen Pflichtethos der Lebensführung tritt der »Leitwert der individuellen Selbstentfaltung « 21 wobei dies nicht nur jeder und jede je für sich selbst beansprucht, sondern genauso den anderen zugesteht, also eine Vielfalt der Lebensweisen toleriert wird.

Für Reckwitz gab es im Golden Age $e^{22}$ eine große, zwar differenzierte, aber doch in sich integrierte »Mittelklasse ", die sich seitdem in drei stark separierte »Klassen « zerlegt habe, von denen eine gar nicht mehr zur »Mitte " gehört: »Während die Dynamik der Postindustrialisierung und der Bildungsexpansion die neue aus der alten Mittelklasse nach oben emporhebt, treiben die gleichen Mechanismen von Postindustrialisierung und Bildungsexpansion nach unten eine neue prekäre

17 In früheren Arbeiten argumentierte Reckwitz (2006) noch rein kulturtheoretisch. Nun verknüpft er - einer langen soziologischen Denktradition folgend - die Wahrnehmung und Bewertung der Gesellschaft, in der jemand sich befindet, mit dessen sozialer Lage.

18 Warum der Begriff der »Spätmoderne" gewählt wurde, erschließt sich zumindest in diesem Text nicht völlig. Marxistische Analysen des »Spätkapitalismus « hatten argumentiert, dass das Arsenal an Mitteln, mit denen der Kapitalismus noch zu retten ist, ausgeschöpft sei (etwa Offe 1972). Reckwitz führt nicht aus, warum und in welchem Sinne nach der "Spätmoderne« die Moderne zu Ende ist, wie der Begriff ja suggeriert.

19 Reckwitz 2019 a, S. 77-85.

20 Ebd., S. 80.

21 Ebd., S. 84.

22 Hobsbawn 1994, S. 324-401. 
Klasse aus der alten Mittelklasse heraus. « ${ }^{23}$ Die »neue Mittelklasse« stellt bei Reckwitz gewissermaßen die Avantgarde gesellschaftlicher Dynamik dar: akademisch gebildet und in zukunftsträchtigen Berufen der Wissensarbeit tätig. Demgegenüber sind die Angehörigen der »prekären Klasse« in der steten Bedrohung, zu den »Überflüssigen « 24 und damit gänzlich abzurutschen; und die »alte Mittelklasse " wird hierzulande zwar noch gesellschaftlich gebraucht, aber gleichsam als schrumpfende Restgröße.

Der Intra-Klassenkompromiss der »nivellierten Mittelstandsgesellschaft« habe diese Zerreißprobe der auseinanderdriftenden Trajektorien nicht überstanden. Stattdessen sei eine sich verschärfende Konfrontation zwischen der »neuen « und der »alten Mittelklasse « entstanden, was sich in den letzten Jahren unübersehbar auch im Zulauf populistischer politischer Gruppierungen zeige. In dieser Konfrontation gehe es um kulturelle Hegemonie. Für die »neue Mittelklasse« dreht sich Reckwitz zufolge das "gute Leben « um »erfolgreiche Selbstverwirklichung ", und »urbaner Kosmopolitismus « wird als wesentliches Charakteristikum einer "guten Gesellschaft " angesehen. ${ }^{25}$ Demgegenüber setzt die »alte Mittelklasse « ein »verwurzeltes Selbst « - hier greift Reckwitz eine Formulierung von Arlie Hochschild ${ }^{26}$ auf - als schon in der "nivellierten Mittelstandsgesellschaft « ausgebildete Leitvorstellung eines "guten Lebens « fort, das sich in einer durch "Sesshaftigkeit " und Wohlgeordnetheit geprägten "guten Gesellschaft« vollziehen soll. ${ }^{27}$ Dieses einstmals kulturell hegemoniale Leitbild ist nun »kulturell in die Defensive geraten $«,{ }^{28}$ verdrängt von den Leitvorstellungen der »neuen Mittelklasse «. In dieser Konfrontation spielt die "prekäre Klasse « keine aktive Rolle; für sie geht es ums "Sichdurchbeißen « in der »Deklassierung «.29 Wichtig ist diese dritte Klasse vor allem als Drohkulisse für die Angehörigen der »alten Mittelklasse«: So wollen sie nicht enden.

Alle drei Klassen sind in Reckwitz' ${ }^{30}$ Einschätzung etwa gleich groß, machen also jeweils knapp ein Drittel der Bevölkerung aus. Dass die »neue Mittelklasse « die kulturelle Hegemonie erlangen konnte und behaupten kann, liegt entscheidend daran, dass eine Koalition von »alter Mittelklasse" und "prekärer Klasse» nicht zustande kommt. ${ }^{31}$ Auch wenn die »alte Mittelklasse« deshalb nicht stark

23 Reckwitz 2019 a, S. 87; Hervorhebung durch die Autoren entfernt.

24 Bude, Willisch 2006.

25 Reckwitz 2019 a, S. 90-96.

26 Hochschild 2017 [2016], S. 166.

27 Reckwitz 2019a, S. 97-102.

28 Ebd., S. 100; Hervorhebung durch die Autoren entfernt.

29 Ebd., S. 102-107.

30 Reckwitz 2019 a, S. 125.

31 Eine Erklärung könnte sein, dass die von Abstiegsängsten geprägte strikte Abgrenzung der »alten Mittelklasse " nach »unten « nicht einmal ein anti-hegemoniales Zweckbündnis zulässt. Und so befände sich die "neue Mittelklasse « - wie man Reckwitz mit einer klassischen Denkfigur Georg Simmels (1968 [1908], S. 89-94) interpretieren kann - in einer stabilen Position des »lachenden Dritten «. 
genug ist, um sich die kulturelle Hegemonie zurückzuerobern, vermag sie es inzwischen doch, der kulturellen Hegemonie der »neuen Mittelklasse " mehr als bloß Nadelstiche zu versetzen. Für Reckwitz drückt sich das gesellschaftspolitisch darin aus, dass das von der »neuen Mittelklasse « bislang favorisierte Politikparadigma des "apertistischen Liberalismus « in die Krise geraten ist und durch einen »einbettenden Liberalismus " ersetzt werden muss, der Leitmotive dessen, was die "alte Mitteklasse" als "gutes Leben " in einer "guten Gesellschaft " begreift, aufnimmt und »erfolgreiche Selbstentfaltung " im »urbanen Kosmopolitismus" dadurch zwar nicht abschafft, aber deutlich relativiert. ${ }^{32}$ Reckwitz sieht eine Chance dafür, dass solch ein »einbettender Liberalismus « »neben dem aufgeklärten und selbstkritischen Teil dieser neuen Mittelklasse auch Teile der alten Mittelklasse sowie der prekären Klasse hinter sich versammeln kann, von denen Teile gegenwärtig zum Populismus abwandern « ${ }^{33}$

Soweit die Grundzüge eines - wie deutlich geworden sein dürfte - in der Verbindung von ungleichheits- und kulturtheoretischer Perspektive nichtreduktionistischen und vieles einzufangen beanspruchenden theoretischen Modells. Unter theorieästhetischen Gesichtspunkten hat Reckwitz hier ein beeindruckendes Kunststück vorgeführt, was sicherlich ein Teil der Erklärung dafür ist, dass die Studie so schnell so viel Aufmerksamkeit gefunden hat. Wir wollen nun aber die darüber hinausreichende Frage stellen: Wie besteht die Analyse den Realitätstest? Das richtet sich nicht nur darauf, ob die vorgelegten theoretischen Argumente zu den empirischen Fakten, soweit sie bekannt und erhärtet sind, passen; auch eventuelle Lücken, Vagheiten und Inkonsistenzen der Argumentation sind hier anzusprechen.

Wir nehmen uns das referierte komplexe Argument im Weiteren zunächst mit Blick auf das Modell der Drei-Klassen-Gesellschaft, dann auf die These eines historischen Bruchs und sodann auf die Schlussfolgerung einer neuen gesellschaftlichen Konfrontationslinie vor.

\section{Drei-Klassen-Gesellschaft?}

Die Grundlage von Reckwitz' Diagnose besteht darin, dass sich zwei Großgruppen in den Mittelschichten herausgebildet haben, von denen sich die eine im Aufstieg und die andere im Abstieg befindet. Das schließt an einen mittlerweile etablierten, wenngleich nicht unumstrittenen Topos in der einschlägigen soziologischen Literatur an. Zu nennen wären für den anglophonen Diskurs zum Beispiel Untersuchungen zur rechtspopulistischen »Tea Party«-Bewegung sowie allgemeiner zu den sogenannten "Culture-Wars" und dem Aufstieg Donald Trumps. ${ }^{34}$ Auch im deutschsprachigen Raum nutzen verschiedene Gegenwartsdia-

32 Reckwitz 2019a, S. 239-304.

33 Ebd., S. 303.

34 Frank 2005; Hochschild 2017 [2016]; Fraser 2017; Kumkar 2017; Kumkar 2018. 
gnosen ein vergleichbares Grundmuster. ${ }^{35}$ In der politikwissenschaftlichen Forschung entspricht dem die Entdeckung der sogenannten »Kommunitaristen «»Kosmopoliten «-Cleavage, ${ }^{36}$ auf die Reckwitz sich ebenfalls bezieht. ${ }^{37}$

Von diesen verwandten Betrachtungen hebt sich Reckwitz' Diagnose durch die interessante und empirisch überprüfbare Zuspitzung ab, dass er den sozialen Raum als eindeutig durch eine Unterteilung in drei und nur drei Klassen strukturiert sieht - eben als Drei-Klassen-Gesellschaft. Er klammert die »Oberklasse" aus, die als das oberste Prozent ${ }^{38}$ nicht nur numerisch zu vernachlässigen sei, sondern auch sonst »über den Dingen « - und damit jenseits des analytischen Interesses - »schwebt «. ${ }^{39}$ Damit verbleiben in seinem Schema nur die »prekäre Klasse", die »neue " und »alte Mittelklasse «, ${ }^{40}$ die jeweils ein Drittel der Gesamtbevölkerung in sich versammeln - eine "Drei-Drittel-Gesellschaft $"{ }^{41}$ So entschieden optiert keine andere der familienähnlichen Theorien. ${ }^{42}$

Vollzieht man Reckwitz' sozialstatistische Interpretationen nach, mit denen er diesen Punkt untermauert, drängt sich allerdings der Eindruck auf, dass die elegante Einfachheit des Schemas mit Uneindeutigkeiten und Widersprüchen in der empirischen Operationalisierung erkauft wird. Sozialstatistisch operationalisiert werden die Klassen entlang der Höhe der Bildungsabschlüsse, des Einkommens, der kulturellen Wertorientierungen und der politischen Selbstverortung. Im Folgenden soll dies entlang der drei erstgenannten Dimensionen, vor allem für die »neue Mittelklasse«, näher betrachtet werden. ${ }^{43}$

35 Kumkar 2015; Nachtwey 2016; Schimank 2018; Koppetsch 2019.

36 Kriesi et al. 2008; Merkel 2017.

37 Wobei Zürn (2020) der eindeutigen Zuordnung der beiden Seiten der Cleavage zu zwei gesellschaftlichen Großgruppen mit jeweils auch darüber hinaus konsistent unterscheidbaren politischen Programmatiken, wie Reckwitz sie hier vornimmt, explizit widerspricht.

38 Reckwitz 2019 a, S. 66.

39 Ebd., S. 107. Eine soziologisch merkwürdige Begründung: als ob das Handeln der Superreichen keine Auswirkungen auf die Lebenschancen aller anderen hätte.

40 Ebd., S. 86.

41 Ebd., S. 125.

42 Pierre Bourdieu (1994 [1979]), dessen Analyse der »feinen Unterschiede « der wichtigste Ausgangspunkt des deutschsprachigen "Zwei Mittelklassen «-Diskurses war, war etwa von einer Sechs-Klassen-Gesellschaft ausgegangen, in der neben den bereits genannten noch das Proletariat, das administrative Kleinbürgertum und ein numerisch durchaus relevantes, wenn auch kleineres Bildungs- und Wirtschaftsbürgertum eine Rolle gespielt hatten.

43 Die politische Dimension ist nicht nur wegen der Schwierigkeiten in der Operationalisierung hier weniger von Interesse. Sie nimmt in Reckwitz' Argumentation, auch wenn er das nicht explizit so rahmt, eher den Status einer abhängigen als einer unabhängigen Variablen ein. Die Verwerfungen entlang der anderen drei Dimensionen sind es, die den politischen Konflikt der Gegenwartsgesellschaft erklären sollen - und nicht, oder zumindest nicht primär, umgekehrt. 
Am eindeutigsten definiert Reckwitz die "neue Mittelklasse « hinsichtlich der Bildungsabschlüsse: Die "neue Mittelklasse" sei eine "Akademikerklasse " ${ }^{44}$ sodass die Feststellung, dass im Jahr 2012 fast jeder Dritte der 30- bis 35-Jährigen in der Bundesrepublik über einen Hochschulabschluss verfügt habe, ${ }^{45}$ auf den ersten Blick eben als Bestätigung der »Drittel-These " gelesen werden kann. So einfach ist es allerdings nicht. Dass in dieser ganze fünf Lebensjahre umfassenden Altersgruppe das Drittel erreicht ist, aber bei allen Älteren nicht, zeigt schon, dass die Gesamtheit noch deutlich unter dem Drittel liegt, Reckwitz’ »Drittel-These « also allenfalls Zukunftsmusik ist.

Neben dieser quantitativen Relativierung seiner These muss man sie in ihrem substantiellen Gehalt ebenfalls zurechtrücken. Auch Reckwitz würde ja vermutlich nicht behaupten, dass ein Hochschulabschluss, gleich welcher Art und welchen Fachs, automatisch die Zugehörigkeit zur »neuen Mittelklasse« bedeutet. Man kann schon fragen, ob nicht mindestens einige klassische Professionen und die Ingenieurswissenschaften ihre Absolventinnen und Absolventen größtenteils auch weiterhin in die gehobene "alte Mittelklasse" entlassen - als Maschinenbauer, Ärzte, Anwältinnen etc. Dasselbe gilt für die Betriebswirtschaftslehre - in Fachhochschulen und Universitäten zusammengenommen das größte Studienfach. Das »konservativ-etablierte " Milieu der SINUS-Studien, das Reckwitz der "alten Mittelklasse « zuordnet, ${ }^{46}$ rekrutiert sich vor allem aus diesen Reihen. ${ }^{47}$ Zieht man diese großen Fächergruppen ab, fällt die »neue Mittelklasse« erheblich kleiner aus.

In der Einkommensdimension ist die Operationalisierung sehr viel uneindeutiger. Reckwitz suggeriert allerdings, dass zumindest tendenziell die Einkommen in der "neuen Mittelklasse " höher als in der "alten Mittelklasse " sind, ${ }^{48}$ was er mit einem Verweis auf den statistischen Zusammenhang von Bildungsabschluss und Einkommenshöhe stützt, wenn er erklärt, dass »die neue Mittelklasse der relative Gewinner der Einkommensentwicklung ist «, weil »[d]ie Daten zeigen: [dass] im Durchschnitt [...] das Einkommen der Akademiker höher als das von Berufstätigen mit mittleren Bildungsabschlüssen ${ }^{49}$ ist. Auch hier verbirgt sich hinter der

44 Reckwitz 2019 a, S. 90.

45 Ebd., S. 81.

46 Ebd., S. 124.

47 Diese Schwierigkeit sieht Reckwitz (ebd., S. 125, Fußnote 67) auch selbst. In unserer Studie war der Fall, der am deutlichsten Reckwitz' Bild der »alten Mittelklasse « zuzuordnen wäre, ein Hochschulabsolvent, der nach abgeschlossener Berufsausbildung Maschinenbau studiert hatte (Holubek-Schaum, Kumkar 2020).

48 Was er allerdings selbst gleich wieder relativiert und veruneindeutigt. So erklärt er mit Bezug auf die neue Mittelklasse: »Das ökonomische Kapital (Einkommen und Vermögen) ist hier durchschnittlich bis überdurchschnittlich « (Reckwitz 2019 a, S. 90) - nur um direkt im Anschluss »eine erhebliche Bandbreite zwischen deutlich überdurchschnittlichem Einkommen und Vermögen [...], durchschnittlichem Mittelklasseeinkommen [...] bis hin zu unterdurchschnittlichem Einkommen [...]« (ebd., S.91) für dieselbe Klasse zu konstatieren.

49 Ebd., S. 88. 
vermeintlichen Eindeutigkeit eine Unschärfe. Denn nicht nur müsste man die gerade erwähnten, oft überdurchschnittlich hoch bezahlten Berufsgruppen der klassischen Professionen, der Betriebswirte und der technischen Berufe mit Hochschulabschluss wieder herausrechnen - was das Einkommensniveau der »neuen Mittelklasse " erheblich nach unten und das der »alten Mittelklasse « entsprechend nach oben korrigieren würde. Es scheint überdies fraglich, ob wirklich alle, die einen mittleren Bildungsabschluss haben, der "alten Mittelklasse" und nicht in relevanten Teilen auch der »prekären Klasse« zuzuordnen sind - was das Einkommensniveau der "alten Mittelklasse" nochmals erhöhte. Beides zusammengenommen könnte ziemlich offen sein, welche der beiden »Mittelklassen « im Schnitt mehr verdient. ${ }^{50}$

Bei der Operationalisierung entlang der Wertorientierung bezieht sich Reckwitz vor allem auf die Ergebnisse der SINUS-Milieu-Studien, deren Milieu-Unterteilung er in sein Drei-Klassen-Modell »übersetzt «. ${ }^{51}$ Diese konzeptionelle Entscheidung überzeugt zunächst, weil mit den Milieus zwei der drei von ihm angesprochenen Dimensionen des Klassenbegriffs, die ökonomische Ressourcenausstattung und die kulturelle Wertorientierung, im Zusammenhang betrachtet werden. ${ }^{52}$ Allerdings wird die vorgenommene »Übersetzung " wenig explizit gemacht und wirft im Resultat Fragen auf, die Reckwitz im Rahmen seines Modells nicht befriedigend beantwortet. Er schlägt das »konservativ-etablierte Milieu«, das »traditionelle Milieu ", die »bürgerliche Mitte" und das "adaptiv-pragmatische Milieu « der "alten Mittelklasse « zu, das »sozialökologische « und das »liberalintellektuelle Milieu « sowie die Milieus der »Performer «, der »Expeditiven « und der »Hedonisten « der »neuen Mittelklasse «. 53 Dass die »Konservativ-Etablierten « traditionelle Werte vertreten, ist zwar qua Definition wahr. Kann man ihr kulturelles Leitmotiv ${ }^{54}$ der »feinen Unterschiede" und besonders der »entre-nousAbgrenzung « nicht ebenso gut als gehobene, connaisseurhafte Form der Ethik der

50 Bezieht man neben dem Einkommen das geerbte Vermögen ein, könnte die "alte Mittelklasse", in der vergleichsweise mehr Personen in die beruflichen Fußstapfen der Eltern treten, noch besser dastehen, während der »neuen Mittelklasse « vorwiegend Personen angehören, die aufgestiegen sind und wenig erben.

51 Ebd., S. 123.

52 Um Reckwitz' Klassenmodell Genüge zu tun, wäre es ja nicht damit getan, zu zeigen, dass der soziale Raum sich entlang der drei Dimensionen dritteln ließe; diese Drittelungen müssten auch zur Deckung kommen. Personen mit akademischem Abschluss müssten also auch ähnliche Werte teilen und sich in einem vergleichbaren und von den anderen beiden Klassen unterscheidbaren Einkommenskorridor bewegen - was Reckwitz aber mit Ausnahme des Verweises auf die SINUS-Milieus nicht empirisch aufzuzeigen versucht.

53 Reckwitz 2019a, S. 123 ff. Wobei er einräumt, dass einige Milieus sich nicht klar zuordnen lassen. Er verortet die Konservativ-Etablierten zwischen »alter « und »neuer Mittelklasse«, das traditionelle Milieu zwischen »alter Mittelklasse« und "prekärer Klasse " und die Hedonisten zwischen »neuer Mittelklasse « und »prekärer Klasse«.

54 Wie es die Definition des SINUS-Instituts bestimmt. In den Definitionen beziehen wir uns, wie Reckwitz auch, auf die Übersicht zu den SINUS-Milieus (www.sinus-institut.d e/sinus-loesungen/sinus-milieus-deutschland/; sowie: www.sinus-institut.de/fileadmin/u 
Singularität verstehen, als deren Träger bei Reckwitz eigentlich die »neue Mittelklasse " gilt? ${ }^{35}$ Beim "adaptiv-pragmatischen Milieu « stellt sich die Frage, ob dessen Offenheit gegenüber kultureller Neuorientierung, die stärker ausgeprägt ist als bei den »Liberal-Intellektuellen « und den »Sozialökologischen « und damit bei der Hälfte der neuen Mittelklasse, es nicht eigentlich eher zum Vertreter der »neuen Mittelklasse « machen sollte. Dass sie laut SINUS-Milieu-Definition ein »Bedürfnis nach Verankerung und Zugehörigkeit" haben, scheint eine eher schwache Begründung dafür, sie als »Kommunitaristen « der »alten Mittelklasse« zuzuschlagen. Andersherum leuchtet nicht unbedingt ein, warum das »sozial-ökologische Milieu «, das kulturellen Neuorientierungen gegenüber wesentlich weniger aufgeschlossen ist und "globalisierungskritische" Positionen vertritt, eindeutig der »neuen Mittelklasse « zuzuordnen ist. Schon mit diesen drei Milieus ist allerdings ein knappes Drittel des sozialen Raums nicht mehr klar den Klassen zuzuordnen.

Zusammengenommen lässt sich festhalten, dass der theoretische Handstreich, der das intuitiv plausible Bild einer gespaltenen »Mittelklasse « zur Diagnose einer »Drei-Drittel-Gesellschaft « verallgemeinert, nur um den Preis erheblicher empirischer Unschärfen möglich scheint. Das soll nun keineswegs bedeuten, dass Reckwitz diese Gruppen und ihre Konflikte einfach »erfunden « hat. Allerdings deutet eben - wie wir in der Folge skizzieren wollen - einiges darauf hin, dass das Bild erheblich differenzierter gezeichnet werden müsste.

Dies könnte auf zwei Weisen geschehen. Auf der einen Seite könnte man im Sinne einer relativierenden Revision von entlang aller drei Dimensionen eher diffusen, ineinander übergehenden Milieus ausgehen, bei denen sich auch die Merkmalsausprägungen entlang der Dimensionen nicht immer im Einklang befinden. ${ }^{56}$ Für ein solches Bild wäre dann allerdings der Begriff der Klasse eindeutig zu stark gewählt; und entsprechend wäre dann auch die Erklärungskraft des Modells in Bezug auf die politischen Konflikte, die Reckwitz im Blick hat, eine deutlich schwächere.

Auf der anderen Seite könnte man im Sinne einer strengen Revision die Klassen genau umgekehrt schärfer gegeneinander abgrenzen, was dann darauf hinausliefe, im sozialen Raum weitere Klassen über die drei von Reckwitz benannten hinaus

ser_data/sinus-institut/Bilder/Sinus-Milieus_092018/2018-09-18_Sinus-Milieus_Websit e_UEberblick_slide.pdf (Zugriffe vom 22.06.2020).

55 Reckwitz scheint dieses Problem auch zu sehen, wenn er diesem Milieu eine neue Form der »Statusinkonsistenz « zuschreibt, die mit seinem hohen, in seinem eigentlichen Klassenschema nicht vorgesehenen kulturellen Kapital einhergeht (Reckwitz 2019 a, S. 125, Fußnote 67). Es stellt sich aber die Frage, ob diese vermeintliche Inkonsistenz nicht ein theoretisch produziertes Problem ist, das sich bei der klassischen bürgerlichen Lebensführung - die akademische Bildung, traditionelle Werte und einen Geschmack der Erlesenheit kombiniert - gar nicht stellt.

56 Auch Reckwitz selbst erklärt ja, dass die Klassen jeweils in Milieus binnendifferenziert sind. Allerdings konstatiert er, dass die Differenzen zwischen den Milieus innerhalb einer Klasse »[...] weniger grundsätzlich sind als jene zwischen den Klassen« (ebd., S. 122). Das wäre erst einmal zu zeigen. Unsere »relativierende Revision « geht davon aus, dass es zahlreiche Milieulagen gibt, die sich nicht klar den jeweiligen Klassen zuordnen lassen. 
zu berücksichtigen. Dafür spräche, dass die Forschung zu Berufsgruppen, Wertorientierungen und Parteipräferenzen ja durchaus gezeigt hat, dass es eine gesellschaftliche Gruppe gibt, in der sich Reckwitz' dreidimensionale Bestimmung der »neuen Mittelklasse « als überdurchschnittlich gebildet bei zumindest mittlerem Einkommen, kosmopolitisch-liberaler Wertorientierung und Präferenz für linke und linksliberale Parteien idealtypisch verwirklicht findet: die sogenannten soziokulturellen Professionen oder »socio-cultural-professions ". ${ }^{57}$ Allerdings ist diese Gruppe, auch wenn sie in den vergangenen Jahrzehnten gewachsen ist, numerisch weit davon entfernt, ein Drittel der Bevölkerung zu umfassen, und ihr gesellschaftlicher Einfluss wäre völlig überschätzt, fasste man sie als »die kulturell, ökonomisch und politisch einflussreichste Gruppe der spätmodernen Gesellschaft ", wie Reckwitz das für die »neue Mittelklasse « behauptet. ${ }^{58}$

Für welche der beiden Revisionen man auch optiert - ob man also von Milieus ausgeht, bei denen ein »harter Kern « von »fließenden Übergängen « umgeben ist, oder ob man von kleineren, eindeutigen Klassenlagen ausgeht, die dann wiederum von anderen Klassenlagen eingefasst sind, die anders bestimmt werden müssten: Beide Konzeptualisierungen bleiben nicht ohne Rückwirkung darauf, wie man die gelebte Erfahrung der »harten Kerne " beziehungsweise eindeutigen Klassenlagen versteht. Denn der von Reckwitz konstatierte Antagonismus zehrt ja von einer Freund-Feind- oder schwächer: »Wir-Andere «-Unterscheidung ${ }^{59}$, die in beiden Konzeptualisierungen wesentlich weniger plausibel wäre. Um im Vokabular der SINUS-Milieus zu bleiben: Ob Personen aus dem »etabliert-konservativen « Milieu sich dem »adaptiv-pragmatischen Milieu « näher fühlen als dem »liberalintellektuellen « oder die "Expeditiven « eher dem »sozial-ökologischen « als dem »adaptiv-pragmatischen Milieu «, erscheint zumindest als empirisch erst zu klärende offene Frage.

Dass die interne Differenzierung der Mittelschichten entweder so gefasst werden könnte, dass sie aus weitaus diffuseren Milieus als Reckwitz' »Klassen « besteht, oder so, dass sie sich in schärfer abgegrenzte, kleinere und zahlreichere "Klassen" gliedert: Darauf verweisen auch die empirischen Befunde aus unserem Forschungsprojekt. Anhand von biografisch-narrativen Interviews haben wir dort die Praktiken der Statusarbeit wie auch die grundlegenden biografischen Orientierungen von Angehörigen der deutschen Mittelschichten rekonstruiert und typisiert und sind dabei zu Ergebnissen gekommen, die systematisch "quer " zu Reckwitz' Überlegungen stehen. Wir waren in der Anlage des Forschungsprojekts davon ausgegangen, bei den Befragten vor allem einen Lebensführungsmodus der »investiven Statusarbeit " rekonstruieren zu können - also überwiegend auf biografische Muster zu stoßen, die sich sowohl alltagspraktisch als auch in der ethisch-sinnhaften Selbstdeutung erkennbar an planvollem, vor allem ökonomischem Statusgewinn ausrichten - flankiert von solchen Fällen, in denen dies zum Beispiel im Sinne einer hedonistischen Statusverweigerung nicht der Fall sei. Dieses Muster

57 Oesch 2008; Oesch, Rennwald 2018; Biskamp 2020.

58 Reckwitz 2019 a, S. 90.

59 Mau 2016. 
wäre, wenn auch in inverser Form, durchaus anschlussfähig an Reckwitz' Diagnose.

Tatsächlich war das Ergebnis der Untersuchung aber ein anderes: Zwar sind zum einen Praktiken der investiven Statusarbeit nicht nur weit verbreitet, sondern für unsere Fälle aus den mittleren und oberen Mittelschichten schlicht unumgänglich. Alle befragten Angehörigen dieser Schichten müssen sich immer wieder mit der Frage auseinandersetzen, wie sie ihren Status planvoll zumindest sichern können. Zum anderen zeigte sich aber, und das ist für die Diskussion des Reckwitz'schen Modells folgenreicher, dass in der Dimension der ethisch-kulturellen Selbstdeutung im Sinne eines biografischen Orientierungsmusters markante Unterschiede auszumachen sind, die wir in einer Typologie aus drei basalen Modi der Lebensführung systematisieren konnten:

(1) Die gemeinschaftszentrierte Lebensführung, in der die Orientierung an Anerkennung durch das unmittelbare soziale Nahfeld dominiert. In dieser Gruppe fanden sich Menschen mit handwerklicher Ausbildung, Facharbeiterinnen und Facharbeiter, aber auch einige mit Hochschulabschluss, vor allem Lehrerinnen und Lehrer. Die Einkommen bewegten sich bei den meisten im Bereich der mittleren Mittelschicht.

(2) Die investive Statusarbeit: Menschen, bei denen vor allem die Orientierung an beruflicher Statusverbesserung biografische Entscheidungen prägt und die ihre sozialen Beziehungen eher distanziert und instrumentell fassen. Auch hier fanden sich Personen mit Hochschulabschluss, eher aus technischen Fächern, und Menschen mit mittlerer Ausbildung; die Einkommen lagen am oberen Rand der mittleren Mittelschicht und in der oberen Mittelschicht.

(3) Die Berufsstolzlebensführung, deren zentrale Orientierung sich auf von Peers und Publikum anerkannte berufliche Meisterschaft ausrichtet, die sie emphatisch als Selbstverwirklichung und -verbesserung verstehen. Auch in anderen Lebensbereichen stellen diese Personen eine Vorliebe für »Besonderes « zur Schau. Diese in unserem Sample sehr kleine Gruppe bestand aus einem Geisteswissenschaftler, einem Musiker und einer Kunsthandwerkerin. Die Einkommen erreichten nicht die Höhe der gutverdienenden investiven Statusarbeiterinnen und -arbeiter, lagen aber höher als bei den meisten, die eine gemeinschaftszentrierte Lebensführung verfolgen.

Nun entspricht die letzte Gruppe ziemlich genau dem Bild der »neuen Mittelklasse ". Allerdings waren die von uns gefundenen Fälle eben auch beruflich in dem schmalen Feld der erwähnten »socio-cultural professions « tätig und könnten daher auch schlicht als jüngste Abkömmlinge des klassischen Bildungsbürgertums verstanden werden. Unsere anderen beiden Typen biografischer Orientierungen unterlaufen demgegenüber Reckwitz' Schema. Die gemeinschaftszentrierte Lebensführung auf der einen Seite ist ohne Frage »sesshaft « und oft auch traditionsverbunden - zeigt aber kein ausgeprägtes Leistungsethos und überdies auch, trotz ihrer relativ zu den anderen beiden Typen oft geringeren ökonomischen Ressourcenausstattung, weder eine starke biografische Verunsicherung noch das Gefühl des »Abgewertetseins «. Auf der anderen Seite haben jene, die sich an investiver Statusarbeit orientieren, das Leistungsethos verinnerlicht - aber diese 
Gruppe ist weder »sesshaft « noch an sozialer Gemeinschaft orientiert, und vor allem ist sie von allen dreien ökonomisch mit Abstand die erfolgreichste.

Man könnte auf zwei Arten versuchen, unsere drei Typen in Reckwitz' Schema zu übersetzen:

- Die eine Möglichkeit wäre, nur die Berufsstolzlebensführung als »neue Mittelklasse « zu fassen. Diese wäre dann sehr viel kleiner ${ }^{60}$ als von Reckwitz angenommen und ökonomisch zwischen zwei Fraktionen der »alten Mittelklasse « angesiedelt, die untereinander aber nicht mehr gemeinsam hätten als mit der »neuen Mittelklasse « - sodass man im Sinne der oben vorgeschlagenen strengen Revision des Klassenschemas auch hier konsequenterweise entscheiden müsste, wer die »eigentliche « "alte Mittelklasse « ist.

- Die andere Möglichkeit bestünde darin, die investiven Statusarbeiterinnen und -arbeiter wegen ihres individualisierten Leistungsethos und ihrer sozialen »Entbettung « der »neuen Mittelklasse« zuzuschlagen - deren Drang nach "Singularisierung « und »Selbstverwirklichung « sich dann allerdings in weiten Teilen in ziemlich traditioneller Statusdemonstration erschöpfen würde. Dies liefe auf die angedeutete relativierende Revision hinaus, an deren Ende man von Klassen eigentlich nicht mehr sinnvoll sprechen könnte.

\section{Bruch?}

Die bis hierher zum Drei-Klassen-Modell artikulierten gewichtigen offenen Fragen und Einwände lassen erwarten, dass auch die beiden gegenwartsdiagnostischen Thesen, die Reckwitz auf Grundlage dieses Modells formuliert, nicht ungeschoren davonkommen. Gibt es tatsächlich einen radikalen Bruch seit Mitte der 1970er Jahre? Und läuft er wirklich auf eine eskalierende Konfrontation von »neuer « und »alter Mittelklasse « hinaus?

Was die These des Bruchs anbelangt, ist zunächst einmal nicht von der Hand zu weisen, dass es gesellschaftsstrukturell seit Mitte der 1970er Jahre durchaus eine ganze Reihe von tiefgreifenden Umbrüchen gegeben hat. Neben den drei von Reckwitz genannten und empirisch gut belegten - Postindustrialisierung, Bildungsexpansion, Wertewandel - lassen sich, ohne Anspruch auf Vollständigkeit, weitere empirisch ebenso unstrittige hinzufügen: Globalisierung, Ökonomisierung, ökologische Krise, Digitalisierung, geopolitische Rearrangements. Jede einzelne dieser Veränderungen und vielfältige Wirkungsverkettungen zwischen ihnen müssten sich eigentlich auch in veränderten Bedingungen der Lebensführung widerspiegeln, wenn denn die soziologische Grundintuition stimmt, dass sich ändernde gesellschaftliche Verhältnisse die Lebensverhältnisse der Gesellschaftsmitglieder tiefgreifend ändern - je nach sozialer Lage natürlich unterschiedlich, aber jeweils mit großer Prägekraft.

60 Selbstverständlich kann unsere qualitative Studie keine Aussagen über Häufigkeiten des Vorkommens eines Typs treffen. Aber: Wenn unsere Rekonstruktion des Zusammenhangs von Berufsfeld und Lebensführung valide ist, dann umfasst der Kern der »neuen Mittelklasse« eben längst nicht alle mit akademischem Abschluss. 
Wir haben bereits gezeigt, dass die Auswirkungen von Postindustrialisierung, Akademisierung und Wertewandel auf die Sozialstruktur vermutlich nicht so einfach zu bestimmen sind, wie Reckwitz dies konstatiert. Blickt man auf die kulturelle Seite der Lebensverhältnisse, also die Modi der Lebensführung in den verschiedenen sozialen Lagen, ist ebenso zu konstatieren, dass die gesellschaftsstrukturellen Wandlungen sich nicht so eindeutig abbilden. Das ist ein noch gewichtigerer Zweifel an Reckwitz' Diagnose, die ja trotz der angestrebten sozialstrukturellen Fundierung im Kern nach wie vor kultursoziologisch angelegt ist. Diesen Zweifel an einem tiefgreifenden Bruch schlüssig zu beweisen dürfte allerdings nicht nur uns schwerfallen. Denn qualitative empirische Studien zu den Lebensführungsmodi der Mittelschichten der 1960er oder 1970er Jahre liegen nicht vor - allein schon deshalb, weil das hierfür benötigte Instrumentarium qualitativer Sozialforschung zu jener Zeit noch gar nicht vorhanden war. Und ersatzweise auf lange, bis in die 1960er Jahre zurückreichende Zeitreihen standardisierter Einstellungs- und Werte-Items zu rekurrieren, wie es am einschlägigsten Ronald Inglehart ${ }^{61}$ getan hat, bietet bestenfalls eine - stets sehr unvollständige Auflistung korrelierter Lebensorientierungen von Personen, lässt aber nicht die Gestalt des Mechanismus, also das Ineinandergreifen der Orientierungen im Wechselspiel mit den Lebenschancen erkennen. ${ }^{62}$

Dennoch können wir - vor allem anhand unserer eigenen Untersuchung - einige Anhaltspunkte dafür benennen, dass Reckwitz auch hinsichtlich eines Umbruchs der Lebensführung reale Veränderungen stark übertreibt. Er schreibt den Mittelschichten der »nivellierten Mittelstandsgesellschaft « als vorherrschenden Lebensführungsmodus "Statusinvestition « zu. ${ }^{63}$ Damit wird unser theoretisches Ausgangsmodell der investiven Statusarbeit aufgegriffen, aber in die Zeit vor dem Bruch eskamotiert. Logischerweise darf dann mindestens eine der heutigen beiden »Mittelklassen « keine investive Statusarbeit mehr betreiben, sondern muss ihre Lebensführung ganz anders ausrichten - sonst gäbe es kulturell keinen Bruch. Diese These ist aber mit Blick auf beide »Mittelklassen « fragwürdig.

Der von ihm für die »neue Mittelklasse « angeführte Lebensführungsmodus der »erfolgreichen Selbstentfaltung « enthält unübersehbar investive Statusarbeit. Es geht um »ein erfolgreiches Leben [...], das mit hohem sozialen Status [...] einher-

61 Inglehart 1977; Inglehart 1998.

62 Reckwitz' eigene empirische Beweisführung vermag nicht zu überzeugen. Er verweist darauf, dass bei den SINUS-Milieus zwischen 1985 und 2018 ein deutliches Anwachsen derjenigen Milieus, die er der »neuen Mittelklasse « zurechnet, zu verzeichnen sei: von insgesamt 13 Prozent auf 31 Prozent (Reckwitz 2019a, S. 125, Fußnote 68). Abgesehen davon, dass die jeweils herangezogenen Milieus teilweise neu auftauchen, teilweise verschwinden und teilweise anders zugeschnitten sind, was einen präzisen Zeitvergleich erschwert: Zahlen für den Zeitraum vor Mitte der 1970er Jahre hat auch Reckwitz nicht. Er vergleicht zwei Zeitpunkte nach dem postulierten Bruch miteinander und verlängert den Anstieg gleichsam nach »hinten «: Weil die besagten Milieus 2018 etwa zweieinhalbmal so groß waren wie 1985, werden sie wohl 1970 nahezu nicht existent gewesen sein. Das ist eine durchaus fragwürdige Schlussfolgerung.

63 Ebd., S. 76-77. 
geht «. ${ }^{64}$ Gemeint ist eindeutig beruflicher Erfolg. Richtig ist zwar, dass für Reckwitz noch etwas zuvor nicht Vorhandenes hinzukommt. Über Erfolg hinaus gelte es auch, »[...] individuelle Wünsche und Begabungen zu entfalten, ein Leben zu führen, das man als befriedigend, sinnvoll und reichhaltig empfindet «. ${ }^{65}$ Die "neue Mittelklasse « kombiniert also Reckwitz zufolge investive Statusarbeit auf der einen, "performative Selbstverwirklichung « in einem " anregenden und erlebnisreichen [...] Leben ", das man vor anderen aufführt und wofür man von diesen soziale Bestätigung erhält, auf der anderen Seite. ${ }^{66}$

Dieses Hinzukommen einer zweiten Leitorientierung der Lebensführung ${ }^{67}$ macht diese zweifellos in sich spannungsreicher, als wenn sich nur alles um investive Statusarbeit dreht. Doch von einem Umbruch des Lebensführungsmodus ließe sich höchstens dann sprechen, wenn beide Leitorientierungen auf "gleicher Augenhöhe " wären, sodass investive Statusarbeit und Selbstentfaltung immer wieder aufeinanderprallen, Lebensführung also die Gestalt eines beständigen Kampfes mit sich selbst annimmt. Das mag es in seltenen Fällen geben; eine solche dauerhafte tiefe innere Zerrissenheit allen Angehörigen der "neuen Mittelklasse" zuzusprechen wäre aber eine äußerst verwegene Behauptung - die Reckwitz auch nicht artikuliert. Er denkt sich diesen Lebensführungsmodus vielmehr so, dass "[...] zwei zunächst feindliche Ideale eine Synthese [...] « eingehen. ${ }^{68}$

Wie das aussehen könnte und ob es plausibel ist, dass dieses Kunststück den meisten Angehörigen der »neuen Mittelklasse « gelingt: Dazu erfährt man bei Reckwitz nicht viel mehr. An manchen Stellen ist er wohl so zu verstehen, dass dieses Kunststück der »neuen Mittelklasse « heutzutage zwar abverlangt werde, sie daran aber regelmäßig scheitere. Reckwitz ${ }^{69}$ konstatiert eine »erschöpfte Selbstverwirklichung " als deren verbreitete Stimmungslage und macht unter anderem ein »Romantik-Status-Paradox" als Ursache aus: "Setzen Individuen beispielsweise radikal auf die Karte Selbstverwirklichung - im Beruf, in der Familie, in der Bildung -, laufen sie Gefahr, dass ihr sozialer Status darunter leidet. Dagegen kann sich bei denjenigen, die fleißig Statusinvestition betreiben und auf Sicherheit setzen, irgendwann das Gefühl einstellen, etwas verpasst oder versäumt $\mathrm{zu}$ haben, die eigenen Potentiale gar nicht ausgelebt zu haben. «70 Auch hier ist wieder empirisch zu fragen: Grassiert wirklich eine flächendeckende »erschöpfte Selbstverwirklichung " in der "neuen Mittelklasse « oder ist das bloß ein Medienhype, den manche sozialwissenschaftlichen Zeitdiagnosen befeuern? Wir haben jedenfalls in unserem empirischen Material bei den wenigen, bei denen ein solcher

64 Ebd., S. 92.

65 Ebd., S. 92.

66 Ebd., S. 217.

67 In früheren Arbeiten spricht Reckwitz (2006, S. 500) auch von einer »ästhetisch-ökonomischen Doublette .

68 Reckwitz 2019 a, S. 210.

69 Ebd., S. 203-238.

70 Ebd., S. 222. 
Orientierungskonflikt biografisch vorkam, herausgefunden, dass dieser in der Regel aufgelöst wurde, indem erwerbsbiografisch auf Statusarbeit umgeschwenkt wurde, die man dann mit »romantischen " Hobbies flankiert - was sicherlich unter den Angehörigen der Mittelschichten auch vor 60 Jahren keine seltene Erscheinung war.

An anderen Stellen legt Reckwitz eine schwächere Lesart der Doppel-Orientierung der »neuen Mittelklasse" nahe. Diese Lesart behauptet eine klare Rangordnung: Selbstentfaltung ist die dominierende biografische Ausrichtung, und beruflicher Erfolg durch investive Statusarbeit wird als nachgeordnetes Mittel zu diesem Zweck eingestuft. So heißt es etwa für die heutige »Spätmoderne«: »[...] die Statusarbeit erweist sich mittlerweile als Rahmenbedingung für gelungene Selbstverwirklichung." Denn investive Statusarbeit verschaffe »jene Ressourcen, die [...] nötig sind, um Selbstentfaltung zu realisieren $«{ }^{71}$ Damit trifft Reckwitz implizit eine Unterscheidung, zu der wir in unserer Untersuchung ebenfalls gelangt sind: zwischen biografischer Ausrichtung auf der einen, Praktiken der so ausgerichteten Lebensführung auf der anderen Seite. Und er behauptet für die »neue Mittelklasse « eine biografische Ausrichtung auf Selbstentfaltung, wofür aber Praktiken investiver Statusarbeit in erheblichem Maße unumgänglich sind.

Etwas Neues ist diese Lesart »erfolgreicher Selbstentfaltung « freilich nicht. Sowohl im Bildungs- als auch im Wirtschaftsbürgertum des 19. Jahrhunderts gab es dies: den Unternehmer, der rastlos und diszipliniert seine Geschäfte betreibt, um mit den Gewinnen eine private Kunstsammlung aufzubauen und in seiner Freizeit als ambitionierter Amateur-Kunsthistoriker mit den Fachleuten auf gleicher Augenhöhe zu kommunizieren. Die heutige Immobilienmaklerin, die spätestens Anfang Fünfzig so viel verdient haben will, dass sie fortan als Rentier ihren eigentlichen Passionen nachgehen kann, setzt dieses Muster nur fort. Dieses Beispiel weist bereits darauf hin, wo Reckwitz' schwächere Lesart zutreffen könnte: in der Oberschicht und allenfalls noch in der oberen Mittelschicht. Keineswegs bringt er damit den in einem Drittel der Gesellschaft gepflegten Lebensführungsmodus zum Ausdruck.

Hinsichtlich der Lebensführung der »neuen Mittelklasse " sehen wir also insgesamt - unterstellt, dass eine dominierende biografische Ausrichtung auf investive Statusarbeit tatsächlich im Golden Age vorherrschte - erhebliche Kontinuitäten und viel geringere Diskontinuitäten als von Reckwitz behauptet. Für seine heutige »alte Mittelklasse " gilt dies ähnlich. Sie ist ja »die unmittelbare Erbin jener einmal allumfassenden Mittelschicht «72 des Golden Age. Auch sie hatte vor Mitte der 1970er Jahre Reckwitz zufolge investive Statusarbeit betrieben, und zwar oftmals erfolgreich in Gestalt sowohl eines je eigenen als auch eines Aufstiegs der

71 Ebd., S. 216-217. Ganz unmissverständlich heißt es bei Reckwitz (2017a, S. 305) unter Bezugnahme auf Schimank et al. (2014), dass in der Lebensführung der »neuen Mittelklasse « »die Statusinvestition nicht Selbstzweck, sondern Mittel zum Zweck der Entfaltung des singularistischen Lebensstils « ist.

72 Reckwitz 2019 a, S. 97. 
eigenen Kinder. Nicht zuletzt aus solchen Aufstiegen innerhalb der Mittelschichten hat sich für Reckwitz das Wachstum der »neuen Mittelklasse « vollzogen.

Mit solchen Erfolgen investiver Statusarbeit auf breiter Front sei es nun allerdings vorbei. Der heutigen »alten Mittelklasse « schreibt Reckwitz überwiegend Merkmale eines Lebensführungsmodus zu, den auch wir in unseren Daten vielfach finden und den wir als gemeinschaftsgeprägt charakterisieren. Solche Personen orientieren ihre Lebensführung in erster Linie daran, in lokalen Gemeinschaften von Verwandtschaft, Nachbarschaft, Freundes- oder Kollegenkreis aufzugehen und sich aufgehoben zu wissen. Dies verbindet sich bei einigen mit dem Stolz auf berufliche Kompetenz und Kompetenzsteigerung, ${ }^{73}$ wozu Reckwitz notiert: »Man entwickelt einen Stolz für die ’harte` (teilweise auch für die ‘gute`) Arbeit, welche dem Leben eine Form gibt. «74

Reckwitz behauptet somit auch hier einen Bruch: einen Wechsel des Lebensführungsmodus dieses Mittelschichtsegments weg von investiver Statusarbeit und hin zu gemeinschaftsgeprägter Lebensführung. In Zeiten der »Neoliberalisierung «, die vielen sozialwissenschaftlichen Diagnosen zufolge den erwerbsfähigen Gesellschaftsmitgliedern ein von Reckwitz ansonsten durchaus auch gesehenes »unternehmerisches Selbst «75 auferlegen, ist das eine überraschende Einschätzung. Plausibel wäre ein solcher Wechsel als unfreiwilliger und resignativer: Weil in der »alten Mittelklasse " inzwischen insbesondere die Ausstattung mit hinreichendem ökonomischen Kapital nicht mehr gesichert ist und das in Gestalt von Bildungstiteln erworbene kulturelle Kapital inflationären Tendenzen unterliegt, vermag man keine irgendwie erfolgversprechende investive Statusarbeit mehr zu betreiben. Doch dieser Fatalismus müsste dann eigentlich - etwa auf der Linie von Oliver Nachtweys ${ }^{76}$ Diagnose der "Abstiegsgesellschaft « - mit massiver Verunsicherung und tatsächlichen sozialen Abstiegen in größerer Zahl einhergehen, was Reckwitz aber als »bislang jedenfalls nicht « gegeben ansieht: »Materiell ist man weiterhin durchaus wohlsituiert oder leidlich versorgt. «77

Mit Blick auf das Golden Age wäre wiederum zu fragen, ob wirklich investive Statusarbeit als dominante biografische Orientierung der damals vorherrschende Lebensführungsmodus der Mittelschichten war. Das könnte ja vielleicht nur für den kleineren Teil der damaligen Mittelschichtangehörigen gegolten haben. Der mehrheitlich gepflegte Lebensführungsmodus - nicht nur der Mittelschichten könnte demgegenüber stark gemeinschaftsgeprägt mit investiver Statusarbeit als nicht allzu anstrengender Zugewinnmöglichkeit gewesen sein. Die meisten wollten womöglich nicht grenzenlos immer weiter aufsteigen: zum einen, weil es irgendwann dann doch mühseliger wurde, bei sinkender Erfolgswahrscheinlichkeit; zum anderen, und wichtiger, wollten sie aus den Gemeinschaften, in denen

73 Diese Lebensführung des Berufsstolzes hat aber ansonsten die stärksten Affinitäten zur Wertorientierung, die Reckwitz der »neuen Mittelklasse « zuschreibt.

74 Ebd., S. 99.

75 Bröckling 2007.

76 Nachtwey 2016.

77 Reckwitz 2019 a, S. 98.

Leviathan, 49. Jg., 1/2021 
sie sich eingelebt hatten, nicht ausbrechen oder herausfallen. ${ }^{78}$ Neben den bereits angeführten lokalen Gemeinschaften, in denen die eigene Lebensführung Anerkennung fand, sind hier auch noch größere gesellschaftliche Milieus wie Arbeiterund Bauernschaft, Kleinbürgertum, Religionsgemeinschaften oder Regionsgemeinschaften wie etwa die Westfalen oder die Rheinländer zu nennen. ${ }^{79}$

Stellen wir dem nun diejenigen unserer empirischen Fälle gegenüber, die Reckwitz' »alter Mittelklasse " entsprechen könnten, zeigt sich auch hier ein komplexeres Bild als das eines klaren Bruchs. Am wichtigsten: Der von Reckwitz angenommene Fortfall investiver Statusarbeit hat nicht stattgefunden. Ein gemeinschaftsoder berufsstolzgeprägter Lebensführungsmodus kann sogar weniger denn je auf sie verzichten - obwohl oder sogar gerade weil sie durch die gesellschaftsstrukturellen Veränderungen anstrengender und erfolgsunsicherer geworden ist. Richtig ist, dass investive Statusarbeit heute bei vielen nicht die biografische Ausrichtung darstellt, sondern nur ein Mittel zu einem anderen Lebenszweck - allerdings ein Mittel, um das niemand herumkommt. Selbst wenn Reckwitz' Portrait der heutigen "alten Mittelklasse" als durch eine gemeinschaftsgeprägte Lebensführung zutreffen sollte, unterschlägt sein Bild doch den Anteil, den Praktiken investiver Statusarbeit auch daran haben. Je weniger aussichtsreich investive Statusarbeit noch mit Blick auf Statusverbesserungen erscheint, desto mehr tritt sie als » Muss « mit Blick auf den bloßen Statuserhalt vor Augen. Hier könnte der eigentliche Unterschied zwischen dem Golden Age und heute liegen. Eine merkliche Veränderung dessen, was man sich von Praktiken investiver Statusarbeit verspricht, wäre das durchaus. Von einem Bruch kann man aber nicht reden, weil mehrheitlich eine Kontinuität der gemeinschaftsgeprägten Lebensführung, wenngleich unter anderen Vorzeichen, gilt.

Damit sehen wir auf beiden Seiten der von Reckwitz behaupteten Bruchkante zu viel Kontinuität, um diese These so aufrechterhalten zu können. Das heißt, um es zu wiederholen, nicht, die für die Lebensführung relevanten Veränderungen und deren Effekte auf die Modi der Lebensführung abzustreiten. Doch dass die

78 Hierfür nur ein Indiz: So sehr Eltern aus der Arbeiterschaft, den unteren oder mittleren Mittelschichten auch begrüßten, dass für ihre Kinder der Weg in die akademische Bildung geöffnet wurde, so sehr wurde besorgt problematisiert, ob sich die eigenen Kinder wohl von ihren Eltern und den Herkunftsgemeinschaften »entfremden « würden, was ja auch tatsächlich in manchen Hinsichten und zeitweise geschah (siehe als plastische Selbstanalyse etwa Eribon 2016 [2009] oder auch die Beobachtungen Bourdieus [1994 (1979), S. 711 f.; 1997; 2002]), bis sich bei vielen entweder Rückbesinnungen auf Herkunftsgemeinschaften oder die Suche nach neuen Gemeinschaften einstellten.

79 Zwar wird das Golden Age auch als diejenige gesellschaftliche Phase angesehen, in der sich bis dahin feste Milieubindungen allmählich aufzulösen begannen - in der Arbeiterund Bauernschaft ebenso wie im Kleinbürgertum; das gleiche galt für Religion und Region als gemeinschaftsstiftende Anker (als Überblick: Kaelble 2007, S. 119-149, 175-206). Doch dass die gesellschaftliche und sozialwissenschaftliche Diskussion dessen, was man dann »Individualisierung « - Ulrich Becks (1983) einschlägige These wurde erst in den 1980er Jahren veröffentlicht und diskutiert - nannte, erst am Ende dieser Ära einsetzte, zeigt, dass diese Anker vielen bis Mitte der 1970er Jahre noch erheblichen Halt geboten hatten. 
Lebensführung der heutigen Mittelschichten in beiden »Klassen " gegenüber dem, wie die Mittelschichten des Golden Age ihr Leben führten, eine völlig umgekrempelte ist, stimmt nicht. ${ }^{80}$

\section{Konfrontation?}

Unsere ausführliche Reflexion der These eines Bruchs hat schon vorbereitet, wie wir uns zu Reckwitz' zweiter These einer zunehmend konfrontativen Beziehung zwischen »alter « und »neuer Mittelklasse « positionieren. Wir können uns daher hier viel kürzer fassen, weil wir nur die diesbezügliche Quintessenz aus dem Bisherigen ziehen müssen.

Für Reckwitz ${ }^{81}$ herrscht ein »Kulturkampf «, der für große Aufgeregtheit in der gesellschaftlichen Mitte sorgt - was so weit gehe, dass Teile der "alten Mittelklasse « anfällig für Rechtspopulismus würden. Die »neue Mittelklasse« sehe sich als Anhängerin einer »Hyperkultur «, die kulturelle Vielfalt als Reservoir für Individualisierung durch »Singularitäten " nutze und mitleidig bis herablassend auf die "alte Mittelklasse" schaue, die in einer kulturelle Grenzen ziehenden Gemeinschaft eine kollektive Identität fände. Umgekehrt halte die »alte« der »neuen Mittelklasse « vor, dass sie aus der Not ihrer »Entwurzelung « durch hemmungslosen Individualismus und Karrierismus eine Tugend mache, der alle nachzustreben hätten, anstatt reumütig in die Gemeinschaft zurückzukehren. Reckwitz stellt hier für beide Seiten heraus, dass sie die je eigene Identität durch Markierung von scharfen - und eindeutig bewerteten - Differenzen herausstreichen.

80 Dafür, dass der - unstrittige - Strukturwandel sich nicht als markanter Bruch in der Lebensführung abbildet, kann man, ohne Anspruch auf Vollständigkeit, mindestens folgende drei Erklärungen anführen. (i) »biographical lag «: Lebensverläufe können den hier und jetzt gegebenen Bedingungen hinterherhinken. Das kann auf materielle Umstände wie etwa Erbschaften zurückgehen. Es kann aber auch um Pfadabhängigkeiten, etwa »Eigensinnigkeiten « der Identitätsbehauptung, oder Bourdieus HysteresisEffekt des Habitus (Bourdieu 1994 [1979], S. 188) gehen. (ii) Schutzzonen: Wer unkündbar im öffentlichen Dienst beschäftigt ist, muss sich vielen Zumutungen der gesellschaftsstrukturellen Umbrüche nicht stellen; und je stärker die wohlfahrtsstaatliche Absicherung ist, desto mehr vermag man diesen Zumutungen zu trotzen (Gottschall et al. 2015). (iii) Komfortzonen: Wer in Berufen mit starker Verhandlungsposition auf dem Arbeitsmarkt tätig ist, kann es sich leisten, seine Lebensführung nicht den nun gegebenen Gelegenheitsstrukturen anpassen zu müssen. Man könnte sagen, dass es sich hier »nur « um Verlangsamungen handelt. Aber erstens ist für alle drei Faktoren durchaus vorstellbar, dass sie nicht bloß übergangsweise, sondern dauerhaft wirken, und dass sie sich nicht auf immer kleiner werdende Teile der Mittelschichten auswirken, sondern auch auf längere Sicht nennenswerte Teile erfassen. Zweitens gilt: Für die Betrachtung dessen, was sich gesellschaftlich - in politischen Kräfteverhältnissen abgebildet - vollzieht, kann es auf dieses Timing entscheidend ankommen. Etwa dafür, ob es - um die wichtigste derzeitige Frage anzusprechen - situative Gelegenheiten für einen anwachsenden Rechtspopulismus gibt. Insofern hängt viel davon $a b$, ob es gelingt, das viel zu grobschlächtige Modell von Reckwitz theoretisch herunterzubrechen und mit empirischen Daten zu füllen.

81 Reckwitz 2019 a, S. 29-61. 
Wenn es aber, wie wir Reckwitz entgegenhalten, mit Blick auf die Grundmuster der Lebensführung keinen tiefen Bruch zwischen der Situation bis Mitte der 1970er Jahre und seitdem gegeben hat, spricht nichts dafür, dass die Mittelschichten sich wie von ihm behauptet intern in zwei konfrontativ gegeneinander aufgestellte Lager auseinanderdividiert haben. Unsere beiden Gegenargumente betonen zum einen strukturelle, zum anderen substantielle Gegebenheiten.

In struktureller Hinsicht geben beide Revisionen von Reckwitz' Gesellschaftsmodell Anlass, nicht von einer gesellschaftsweit dominanten und prägenden Zwei-Klassen-Frontlinie auszugehen. Die relativierende Revision besagt, dass es keine schroffen Grenzen zwischen den beiden von Reckwitz unterschiedenen Klassen gibt, sondern fließende Übergänge, die Differenzen und mögliche darin angelegte Konflikte moderieren. Zwischen den »Streithähnen" beider Klassen befindet sich eine Überzahl von mindestens ambivalenten Gesellschaftsmitgliedern, die sich - schon um ihre »Ruhe « zu haben - ganz aus dem Streit heraushalten. Die strenge Revision muss davon ausgehen, dass es neben dem, was Reckwitz "alte" und "neue Mittelklasse " nennt, noch eine größere Zahl weiterer Klassen wenn der Begriff dann überhaupt noch passt - gibt. Dass diese sich alle von Reckwitz' beiden Antipoden entweder auf die eine oder die andere Seite ziehen lassen, ist zwar prinzipiell nicht ausgeschlossen, müsste aber empirisch erst einmal gezeigt werden.

Wahrscheinlicher ist zunächst, dass all diese anderen sozialen Lagen ihre je eigenen Interessen und Interessenkonflikte untereinander und mit Reckwitz' Klassen haben, sodass sich insgesamt ein viel zerklüfteteres Bild von »Kampfzonen « bietet. Wenn man so will, ist das ja einer der grundlegenden Mechanismen gesellschaftlicher Sozialintegration in der Moderne: Vervielfältigung von Konfliktfronten, sodass sich - um die marxistische Terminologie zu übernehmen - der eine »Hauptwiderspruch « in beliebig viele "Nebenwidersprüche « auflöst, deren ordnungsgefährdendes Potenzial sich in Grenzen hält. Dass dieser Mechanismus durch die gesellschaftlichen Strukturdynamiken der letzten Jahrzehnte nicht bloß geschwächt, sondern vielleicht ganz außer Kraft gesetzt worden ist, ist eine weitere gewagte Unterstellung, die zu begründen wäre, wollte man diese Lesart der Konfrontations-These aufrechterhalten.

In substantieller Hinsicht stellen wir fest, dass aus den von uns gefundenen biografischen Orientierungen kein inhärent angelegtes schroffes Gegeneinander erwächst. Weder jene, die sich an investiver Statusarbeit orientieren, noch jene, die der gemeinschafts- oder berufsstolzgeprägten Lebensführung anhängen, sind aus der Logik des jeweiligen Lebensführungsmodus heraus zwangsläufig auf Konfrontationskurs mit anderen Modi. »Gute « investive Statusarbeit konstituiert sich nicht primär darüber, dass man sich verächtlich gegenüber denjenigen abgrenzt, die gemeinschaftsgeprägt leben, und umgekehrt - und das Gleiche gilt für eine berufsstolzgeprägte Lebensführung. Jeder der Modi begründet sich aus sich selbst heraus, nicht als unversöhnlicher Antipode von etwas anderem.

Wir sind denn auch in unserer Untersuchung auf keine durchgängig die Selbstverortung formatierende konfrontative Rhetorik gestoßen, mit der Angehörige der "alten« die »neue Mittelklasse» abwerten und umgekehrt. Weder finden sich 
häufiger Äußerungen, die einen Backlash der alten, sich auf der Verliererstraße wähnenden kulturellen Hegemonie zum Ausdruck bringen. ${ }^{82}$ Noch gibt es auftrumpfende Stellungnahmen derer, die den "Fortschritt" auf ihrer Seite sehen. Einzelne Bemerkungen, in denen etwa »Akademiker « als »ewige Studenten «, die sich vor der Arbeit drücken, bezeichnet oder die altbekannten Stereotype in der anderen Richtung geäußert werden, gehen meist auf persönliche Konstellationen, etwa Konkurrenzsituationen zwischen Geschwistern, zurück, auch wenn sich damit mitunter durchaus generalisierende Milieubewertungen verbinden. Wenn solche kulturellen Differenzen angesprochen wurden, fanden wir eher Nachdenklichkeit vor, die alles andere als eine selbstbewusste oder gar selbstgerechte Propagierung der eigenen Lebensprinzipien für alle zum Ausdruck bringt. Nicht mal diejenigen unserer Fälle, die Reckwitz' beiden Mittelklassen ganz eindeutig zuzuordnen wären, befleißigen sich konfrontativer Rhetorik - ganz zu schweigen von der großen Mehrzahl derjenigen Fälle, die nicht so klar in dieses Raster fallen.

Aufschlussreich ist hier auch: Wo immer wir Statusdemonstration beobachten konnten, was vor allem - aber nicht nur - bei den investiven Statusarbeitern der Fall war, blieben unsere Interviewten in ihrer Zurschaustellung des eigenen Statuserfolgs äußerst konventionell. ${ }^{83}$ Die Statusdemonstration soll gar nicht über »Singularitäten « individuelle Einzigartigkeit zum Ausdruck bringen, sondern ganz traditionell anderen damit imponieren, dass man mehr von etwas hat, was sie auch alle für begehrenswert halten - etwa teure Autos von bekannten Edelmarken oder Fernreisen zu den allseits bekannten Orten, die man »sich leisten können « muss. Das bleibt den kulturellen Maßstäben der »alten Mittelklasse « verhaftet, die also in der Statusdemonstration als Referenzrahmen und nicht etwa zur Abgrenzung herangezogen wird. Wohlgemerkt: Wir behaupten auch an diesem Punkt nicht, dass es das Milieu des Prenzlauer Bergs - um das Klischee zu zitieren - gar nicht gibt. Doch es gibt daneben, und vermutlich zahlenmäßig überwiegend, viele Mittelschichtangehörige, die sich in den Portraits, die Reckwitz von der »alten " und der »neuen Mittelklasse « zeichnet, nicht wiederfinden dürften und die auch in der damit verbundenen, doch angeblich die Dynamik der gesamten Gesellschaft prägenden Konfrontation zwischen diesen beiden Klassen keine klare Position beziehen würden.

82 Diesen Backlash sehen Ronald Inglehart und Pippa Norris (2016) als zentrale Erklärung des erstarkenden Rechtspopulismus an. Siehe weiterhin die von Arlie Hochschild (2017 [2016]) in den USA eingefangenen Stimmungsbilder aus Reckwitz' »alter Mittelklasse «.

83 Es sei allerdings angemerkt, dass Praktiken der Statusdemonstation nicht im Zentrum der Untersuchung standen. Die prägnantesten Beispiele »passierten einfach so « entweder in der Interviewsituation selbst, etwa in Bezug auf das Wohnambiente, oder indem die Interviewten Statusdemonstration in Situationsschilderungen im Rahmen ihrer biografischen Stegreiferzählung berichteten. 


\section{Schluss}

Reckwitz hat sich mit dem »Ende der Illusionen « an eine Aufgabe herangewagt, die sowohl aufgrund gesellschaftlicher Entwicklungen als auch aufgrund gesellschaftstheoretischer Fortentwicklungen auf die Tagesordnung gekommen ist. Er legt ein Gesellschaftsmodell vor, das nichtreduktionistisch und auch nicht einfach bloß eklektizistisch eine kultur- und eine ungleichheitstheoretische Perspektive auf die »Spätmoderne « zusammenführt. Wenn wir hier eine längere Liste von Mängeln vorlegen, soll das keineswegs die Schlussfolgerung nahelegen, dass man Reckwitz' Modell nun sang- und klanglos ad acta legen sollte. Es muss im Gegenteil weiter in der Diskussion bleiben. Denn selbst wenn sich herausstellen sollte, dass der theoretisch weite Bogen, den Reckwitz spannt, den Stresstest der empirischen Konkretisierung nicht heil übersteht, dann verweist gerade das auf Bruchstellen im kategorialen Gerüst, deren Kenntnis für eine anschließende Konstruktion vergleichbar umfassender zeitdiagnostischer Überlegungen von kaum zu überschätzendem Wert sein dürfte.

Je weniger sich als substantiell bewahrenswert erweist, desto mehr muss man sich allerdings von einer weiteren Nutzanwendung des Modells verabschieden, die auch zu seiner Attraktivität beigetragen hat. Reckwitz leitet aus seinem Modell ja auch eine Erklärung dafür ab, warum in den letzten Jahren in Deutschland wie in vielen anderen Ländern Europas und Nordamerikas ein Rechtspopulismus angewachsen ist und inzwischen zu einer nicht länger vernachlässigbaren politischen Größe geworden ist. Die "prekäre Klasse", aber auch die »alte Mittelklasse" erweisen sich Reckwitz zufolge als zunehmend anfällig für politische Kräfte wie in Deutschland die AfD, und zwar aufgrund ihres tatsächlich erfolgten oder befürchteten sozialen Abstiegs - sowohl in ökonomischer Hinsicht als auch als kulturelle Degradierung ihrer Lebensweise. In dem Maße, in dem sich Reckwitz' Charakterisierung dieser beiden Klassen in den von uns kritisierten Punkten nicht halten lässt, fällt seine Interpretation des Rechtspopulismus in sich zusammen. Wir schicken uns hier nicht an, sogleich eine bessere Alternative aus dem Ärmel zu schütteln - allein schon, weil die Prüfung von Reckwitz' Modell noch nicht abgeschlossen ist. Aber wir möchten auf diese wichtige Implikation schon jetzt aufmerksam machen.

Schließlich wird sich, je weniger das ursprüngliche Modell sich als haltbar erweisen sollte, die Frage, warum es solch eine begeisterte Rezeption durch Feuilleton und Politik erfährt, nochmals in anderem Licht stellen. Die Antwort auf diese Frage kann dann ja nicht mehr einfach lauten: weil es stimmt. Man wird vielmehr eine wissenssoziologische Antwort suchen: Was an dem Modell bedient - ohne dass man Reckwitz dabei irgendeine Intention unterstellen muss oder sollte - welche Interessen von Journalistinnen und Journalisten, Aufmerksamkeit zu erlangen, und von Politikerinnen und Politikern, sich als »Wähler-Versteher « zu profilieren? Wir werden zu dieser Frage in einem weiteren Beitrag gesondert eine Antwort zu geben versuchen. 


\section{Literatur}

Balzter, Sebastian; Bernau, Patrick; Weiguny, Bettina 2020. »In Corona-Zeiten: Chefs im Homeoffice", in FAZ.NET vom 13. April 2020. www.faz.net/1.6722711 (Zugriff vom 22.06.2020).

Beck, Ulrich 1983. "Jenseits von Stand und Klasse", in Soziale Ungleichheiten, hrsg. v. Kreckel, Reinhard, S. 35-74. Göttingen: Schwartz.

Biskamp, Floris 2020. »Kein Kommunitarismus, nirgends. Eine Retraditionalisierung wird die Sozialdemokratie nicht retten. Replik auf Carsten Nickel «, in Leviathan 48, 1, S. 70-89.

Bourdieu, Pierre 1994 [1979]. Die feinen Unterschiede: Kritik der gesellschaftlichen Urteilskraft. Frankfurt a. M.: Suhrkamp.

Bourdieu, Pierre 1997. »Widersprüche des Erbes", in Pierre Bourdieu: Das Elend der Welt: Zeugnisse und Diagnosen alläglichen Leidens an der Gesellschaft, S. 651-658. Konstanz: UVK.

Bourdieu, Pierre 2002. Ein soziologischer Selbstversuch. Frankfurt a. M.: Suhrkamp.

Bröckling, Ulrich 2007. Das unternehmerische Selbst. Soziologie einer Subjektivierungsform. Frankfurt a. M.: Suhrkamp.

Bröckling, Ulrich 2019. »Dialektik der Modernisierung: Rezension zu >Das Ende der Illusionen von Andreas Reckwitz «, in Soziopolis (blog) vom 16. Dezember 2019. www.soziopolis.de/ lesen/buecher/artikel/dialektik-der-modernisierung/ (Zugriff vom 22.06.2020).

Bude, Heinz; Willisch, Andreas. Hrsg. 2006. Das Problem der Exklusion. Ausgegrenzte, Entbehrliche, Überflüssige. Hamburg: Hamburger Edition.

Buroway, Michael 2005. "For Public Sociology ", in American Sociological Review 70, 1, S. 4 28.

Charim, Isolde 2018. »Knapp überm Boulevard - Eine Klasse für sich und eine Klasse gegen sich ", in die tageszeitung vom 27. November 2018.

Eribon, Didier 2016 [2009]. Rückkehr nach Reims. Berlin: Suhrkamp.

Frank, Thomas 2005. What's the Matter with Kansas? How Conservatives Won the Heart of America. New York: Holt Paperbacks.

Fraser, Nancy 2017. "From Progressive Neoliberalism to Trump - and Beyond «, in American Affairs 1, 4, S. 46-64.

Goffman, Erving 1959. The Presentation of Everyday Life. New York: Doubleday.

Gottschall, Karin; Kittel, Bernhard; Briken, Kendra; Heuer, Jan-Ocko; Hils, Sylvia; Streb, Sebastian; Tepe, Markus 2015. Public Sector Employment Regimes - Transformations of the State as an Employer, Transformations of the State. Houndmills, Basingstoke: Palgrave Macmillan.

Grau, Alexander 2019. "So weltoffen, so borniert! «, in Der Tagesspiegel vom 24. November 2019.

Hobsbawn, Eric 1994. Das Zeitalter der Extreme. Weltgeschichte des 20. Jabrhunderts. München: Hanser.

Hochschild, Arlie Russell 2017 [2016]. Strangers in Their Own Land: Anger and Mourning on the American Right. New York, London: The New Press.

Holubek-Schaum, Stefan; Kumkar, Nils C. 2020. »Der >Dämon`, der des >Lebens Fäden` hält. Konzeptionelle Überlegungen zum Stellenwert des Statusbegriffes in der Lebensführungsforschung ", in Transformationen alläglicher Lebensführung. Konzeptionelle und zeitdiagnostische Fragen, hrsg. v. Jochum, Georg; Jurczyk, Karin; Voß, Günther G.; Weihrich, Margit, S. 152-169. Weinheim, Basel: Beltz Juventa.

Inglehart, Ronald 1977. The Silent Revolution. Changing Values and Political Styles Among Western Publics. Princeton: Princeton University Press.

Inglehart, Ronald 1998. Modernisierung und Postmodernisierung. Kultureller, wirtschaftlicher und politischer Wandel in 43 Gesellschaften. Frankfurt a. M.: Campus.

Inglehart, Ronald; Norris, Pippa 2016. Trump, Brexit, and the Rise of Populism. Economic Have-Nots and Cultural Backlash. HKS Faculty Research Working Paper Series 16-026. Cambridge: Harvard Kennedy School.

Kaelble, Hartmut 2007. Sozialgeschichte Europas. 1945 bis zur Gegenwart. München: C. H. Beck. 
Koppetsch, Cornelia 2019. Die Gesellschaft des Zorns: Rechtspopulismus im globalen Zeitalter. Bielefeld: transcript.

Kriesi, Hanspeter; Grande, Edgar; Lachat, Romain; Dolezal, Martin; Bornschier, Simon; Frey, Timotheos 2008. West European Politics in the Age of Globalization. Cambridge: Cambridge University Press.

Kumkar, Nils C. 2015. "»... als ob man von Bratenduft satt werden könnte< - Der Diskurs der AfD am Beispiel der Gründungsparteitagsrede von Bernd Lucke", in Rechtsextremismus der Mitte und sekundärer Autoritarismus, hrsg. v. Decker, Oliver; Kiess, Johannes; Brähler, Elmar, S. 127-150. Gießen: Psychosozial-Verlag.

Kumkar, Nils C. 2017. »Autoritarismus und Realitätsverlust: Das Krisenerleben des klassischen Kleinbürgertums und die Attraktivität Donald Trumps " in Psychologie und Gesellschaftskritik 41, 3/4, S. 87-107.

Kumkar, Nils C. 2018. The Tea Party, Occupy Wall Street, and the Great Recession. Basingstoke, New York: Palgrave Macmillan.

Mau, Steffen 2012. Lebenschancen. Wohin driftet die Mittelschicht? Frankfurt a. M.: Suhrkamp.

Mau, Steffen 2016. »Ungleichheit ist längst kein Monopol der Linken mehr ", in Frankfurter Allgemeine Sonntagszeitung vom 25. September 2016.

Merkel, Wolfgang 2017. "Kosmopolitismus versus Kommunitarismus. Ein neuer Konflikt in der Demokratie", in Parties, Governments, and Elites: The Comparative Study of Democracy, hrsg. v. Harfst, Philipp; Kubbe, Ina; Poguntke, Thomas, S. 9-23. Wiesbaden: Springer VS.

Nachtwey, Oliver 2016. Die Abstiegsgesellschaft: Über das Aufbegehren in der regressiven Moderne. Berlin: Suhrkamp.

Nassehi, Armin 2020. "Selbstverwirklichung ist anstrengend «, in Frankfurter Allgemeine Zeitung vom 18. Februar 2020.

Oesch, Daniel 2008. "The Changing Shape of Class Voting ", in European Societies 10, 3, S. 329-355.

Oesch, Daniel; Rennwald, Line 2018. „Electoral Competition in Europe's New Tripolar Political Space: Class Voting for the Left, Centre-Right and Radical Right ", in European Journal of Political Research 57, 4, S. 783-807.

Offe, Claus 1972. »Spätkapitalismus - Versuch einer Begriffsbestimmung «, in Claus Offe: Strukturprobleme des kapitalistischen Staates, S. 7-25. Frankfurt a. M.: Suhrkamp.

Pausch, Robert 2020. »Heldchen der Arbeit «, in DIE ZEIT vom 9. Januar 2020. www.zeit.de/ 2020/03/fdp-christian-lindner-liberalismus?utm_referrer=https $\% 3 \mathrm{~A} \% 2 \mathrm{~F} \% 2 \mathrm{Fwww}$.google. de\%2F (Zugriff vom 10.12.2020).

Reckwitz, Andreas 2006. Das hybride Subjekt. Eine Theorie der Subjektkulturen von der bürgerlichen Moderne zur Postmoderne. Weilerswist: Velbrück Wissenschaft.

Reckwitz, Andreas 2017 a. Die Gesellschaft der Singularitäten. Zum Strukturwandel der Moderne. Berlin: Suhrkamp.

Reckwitz, Andreas 2017 b. »Anklage? Analyse!«, in Süddeutsche Zeitung vom 22. September 2017.

Reckwitz, Andreas 2019 a. Das Ende der Illusionen. Politik, Ökonomie und Kultur in der Spätmoderne. Berlin: Suhrkamp.

Reckwitz, Andreas 2019b. »Liberalismus: Ein Ordnungsruf «, in DIE ZEIT vom 16. November 2019. www.zeit.de/2019/47/liberalismus-regulierungen-dynamisierung-demokratie-nat ionaloekonomie (Zugriff vom 22.06.2020).

Reckwitz, Andreas 2020. "Kampf um das Bürgerliche", in Der Spiegel 8 vom 15. Februar 2020, S. 116-119.

Reinecke, Stefan 2020. »Im Kollektiv zu denken fällt manchen schwer . Interview mit Andreas Reckwitz ", in die tageszeitung vom 23. März 2020.

Sauerbrey, Anna 2020. "Keiner hortet Sushi. Was die Krise mit der hyperindividualisierten Gesellschaft macht", in Der Tagesspiegel vom 22. März 2020.

Schimank, Uwe 2018. »Rechtspopulistische Mittelschichten als Gefährder gesellschaftlicher Ordnung: Eine theoretische Skizze", in Die Mitte als Kampfzone: Wertorientierungen und Abgrenzungspraktiken der Mittelschichten, hrsg. v. Schöneck, Nadine M.; Ritter, Sabine, S. 217-240. Bielefeld: transcript. 
Schimank, Uwe; Mau, Steffen; Groh-Samberg, Olaf 2014. Statusarbeit unter Druck? Zur Lebensführung der Mittelschichten. Weinheim, Basel: Beltz Juventa.

Schlieben, Michael 2020. »Friedrich Merz: ,Wir können in dieser Krise nicht allen helfen «, in ZEIT online vom 2. Mai 2020. www.zeit.de/politik/deutschland/2020-05/friedrich-merz-c oronavirus-politik-wissenschaft-massnahmen-pandemie?utm_referrer $=\mathrm{https} \% 3 \mathrm{~A} \% 2 \mathrm{~F} \% 2 \mathrm{~F}$ www.google.de\%2F (Zugriff vom 10.12.2020).

Schütz, Simon; Schuler, Ralf 2020. »Lindner, Merz und Klingbeil lesen dieselbe Lektüre. Das Geheimnis hinter diesem Buch", in BILD vom 3. April 2020.

Simmel, Georg 1968 [1908]. Soziologie. Untersuchungen über die Formen der Vergesellschaftung. Berlin: Duncker \& Humblot.

Sobocynski, Adam 2020. »Armin Nassehi und Andreas Reckwitz: Zwei Soziologen und die Leitkultur «, in DIE ZEIT vom 20. Februar 2020. www.zeit.de/2020/09/leitkultur-armin-n assehi-andreas-reckwitz-soziologie (Zugriff vom 10.12.2020).

Teuwsen, Peer 2020. » Am Ende ist das Leben verarmt< - Ein Gespräch mit dem Soziologen Andreas Reckwitz«, in Neue Zürcher Zeitung am Sonntag vom 3. Mai 2020.

Wohlfahrt, Tom 2020. »Im Kreuzfeuer ", in die tageszeitung vom 11. Januar 2020.

Zürn, Michael 2020. "Zurück zur Sozialistischen Internationale? Replik auf Carsten Nickel, Floris Biskamp und Michael Hartmann «, in Leviathan 48, 2, S. 161-175. 
Zusammenfassung: Andreas Reckwitz hat kürzlich mit dem Buch Das Ende der Illusionen (2019) eine einflussreiche Diagnose der aktuellen gesellschaftlichen Situation und ihrer Zukunftsperspektiven vorgelegt, die von uns einer kritischen Würdigung unterzogen wird. Die Attestierung theoretischer Eleganz geht mit einer längeren Mängelliste hinsichtlich der empirischen Haltbarkeit zentraler Thesen einher.

Stichworte: Gegenwartsdiagnose, Mittelschichten, Lebensführung, Kultur, Rechtspopulismus

\title{
Three-Class Society? Historical Break? Confrontation? A Dispute with Andreas Reckwitz’ Diagnosis of "Late Modernity «
}

Summary: With his book The End of Illusions (2019), Andreas Reckwitz recently presented an influential diagnosis of the current social situation and its future prospects. Our critical reading points out - despite theoretical elegance - a long list of empirically doubtful claims.

Keywords: diagnosis of the present, middle classes, conduct of life, culture, right-wing populism

\author{
Autoren \\ Nils Kumkar \\ Universität Bremen \\ SOCIUM - Forschungszentrum Ungleichheit und Sozialpolitik \\ Mary-Somerville-Str. 9 \\ 28359 Bremen \\ Deutschland \\ kumkar@uni-bremen.de \\ Uwe Schimank \\ Universität Bremen \\ SOCIUM - Forschungszentrum Ungleichheit und Sozialpolitik \\ Mary-Somerville-Str. 9 \\ 28359 Bremen \\ Deutschland \\ uwe.schimank@uni-bremen.de
}

